\title{
Physical Activity Practitioners: Reducing Infection with Coronavirus
}

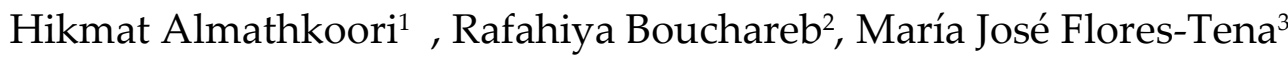

\section{ARTICLE INFO}

Article History:

Received 06.03.2020

Received in revised form

16.06.2020

Accepted

Available online 01.10.2020

\begin{abstract}
Physical activity is carrying out a conscious voluntary physical effort, as the body gains health and protection from diseases. There are many different types of physical activities. It is possible to do walking, running, jumping, climbing, etc. These matters are important in a person's life, so if the individual does not like a type Among them, there are many options, and there are many games that are practiced as a kind of entertaining sport such as football, basketball, and small games. Therefore, everyone must set hours per day to exercise, especially during the home ban that results in lack of movement, excessive eating, and more sleep with The possibility of weight gain and thus the high risk of developing serious and chronic diseases such as: diabetes, high blood pressure and arthritis, in addition to mental illnesses such as depression, anxiety, fatigue and stress, which in turn leads to psycho-motor disorders. The researchers used a questionnaire that was distributed to 80 persons from the Algerian community, whose ages ranged from 45-55 years, during which a set of questions was asked and the answers were closed in the selection of them chosen by the researchers, and the most important findings of the researchers in their study is that physical activity and exercise within the limits The house contributes effectively to feeling healthy and safe from diseases, improving the recreational side and avoiding psychological pressures.
\end{abstract}

(C) IJERE. All rights reserved

Keywords:

Physical sports activities, coronavirus, public health.

\section{INTRODUCTION}

Exercise is a subcategory of PA that is planned, structured, and repetitive and that has as a final or intermediate objective for improvement or maintenance of physical fitness. Physical function is the capacity of an individual to perform the physical activities of daily living. Physical function reflects motor function and control, physical fitness, and habitual PA (Langhammer, Bergland and Rydwik, 2018). There are many different types of physical activities. Walking, running, jumping, climbing and other things can be done in a person's life. He does not like a type of it, he has many options, and there are many games that are practiced as a kind of entertaining sport such as football, basketball, and small games. Therefore, everyone must limit hours per day to exercise, especially during the home ban that results in lack of movement and eating excessive and a large number of sleep with the possibility of weight gain and thus an increased risk of developing serious and chronic diseases such as: diabetes, high blood pressure and arthritis, in addition to psychological diseases such as depression, anxiety, boredom and tension, which in turn leads to psychomotor disorders.

Exercising on a daily basis burns the extra calories in the body and burns the accumulated fat as the body excretes it in the form of sweat. Many people are lazy or afraid to start a physical training program, but it should not be awe. The best way to get started is to look at your schedule and decide where to regularly exercise a 30-minute exercise. And many normal daily activities are considered moderate exercise, for example: climbing stairs, walking on a moving mat, or playing with children, and physical activity does not necessarily take place in one session, you can distribute 30 minutes - for example, by walking for a period of 10 minutes, then do the running for 20 minutes later, for example with children. Research has shown that the division of physical activity for ten minutes throughout the day burns calories no less than the number of calories burned over a continuous period of time. In this regard, the results of a qualitative study than in the United Kingdom for the export of overweight individuals, as well as health professionals and policy makers working in the field of obesity prevention and weight management (Greener, Douglas,Edwinvan and Teijlingen, 2010). Once people engage in the habit of allocating time on most days of the week for physical activity, they tend to be more open to new types of activities - because they feel stronger and more able to exercise. People who have not thought of a new sport may find themselves inclined to experience dancing,

1 dr.hikmat6@yahoo.com, Almustansiriyah University/Iraq, orcid.org/ 0000-0002-7103-203X

2 rafahiya.bou@gmail.com, University of Batna /Algeri, orcid.org/ 0000-0001-9491-3350

3 maria.flores@uam.es, Universidad Autónoma de Madrid, orcid.org/ 0000_0001-8293-9043 
or entertaining exercises, and then they become more active over time, which helps them treat weight gain (Nuñez-Gonzalez, et al. 2017 ). Being active doing activities improves the quality of life of people having a greater well-being in health. The world health organization reports that at least $60 \%$ of the world population does not carry out the physical activity necessary to obtain health benefits (Flores-Tena, 2019). Participation in PA and exercise can contribute to maintaining quality of life, health, and physical function and reducing falls ( Gillespie, Robertson and Gillespie, 2012). Among older people in general and older people with morbidities in particular. The increased attention to the relationship between exercise and HRQOL in older adults over the last decade is reflected in a recent review, which showed that a moderate PA level combining multitasking exercise components had a positive effect on activities in daily living, highlighting the importance of physical, mental, and social demands (Langhammer, 2018).

Exercise works to relieve stress, depression and negative energy from the body, and these feelings affect the heart and its health. Exercise also increases the body's ability to sleep and drown in it, thus allowing the heart muscle to relax a little from the workload. The body's activity during sleep is a little compared to the day and during work, and it also lowers blood pressure for those suffering from hypertension. High level of happiness and improvement of mood in humans, and this works to stimulate the heart muscle and protect it from diseases and to accustom the heart muscle to the high effort and thus increase the efficiency of its work and not feel fatigue and fatigue when making any sudden effort. But you must stay away from strenuous exercise in cases of heart disease due to lack of muscle strain, such as: mountaineering, and speedy running, but fast walking can be practiced with enough rest from time to time and stop playing in case of extreme fatigue or Feeling of shortness of breath. Several previous studies had reached similar results about physical activity and cancer, but in the last analysis, the researchers conducted deeper studies on the relationship between the level of physical activity and how to reduce the risk of cancer. We take an example of breast cancer, as some recent studies have shown that continuous sports activity helps reduce the risk of developing breast cancer. The risk of relapse is also not high in women who have physical and athletic activity hours per week. A physical inactivity generates 3.2 million deaths worldwide, it is the fourth risk factor for global death, it is the main cause of approximately between 21 to $25 \%$ of breast and colon cancers, $27 \%$ of cases of diabetes and approximately $30 \%$ of the burden of ischemic heart disease. Sports physical activity and its relationship to raising the average age, Studies have shown that continuing to exercise after reaching the age of 40 extends life for a period ranging between two and seven years. The American Health Day News website reported that the American National Cancer Institute prepared a study during which it became clear that staying active after 40 years of age increases between two and seven years of age, and the study also showed that the more exercise one performs, the longer his life regardless of his weight." There is a close relationship between sport and age, and if you are not doing any sporting activity now, you should know that a few minutes a day may significantly increase your age," said Stephen Moore, a study participant $(\mathrm{WHO}, 2020)$. Carrying out a physical activity that increases the secretion of endorphins in the blood, and that makes people permanently renewed, making them love life and accept it happily. Endorphins may be one of the most known hormones in the general contexts between humans. It is produced by the pituitary gland, and it is known mainly as an anti-pain. The most important stimuli for endorphins in our bodies is the practice of strenuous physical activities to the point of ecstasy. This justifies the recommendations to maintain a healthy activity and healthy regular life. In addition to acupuncture. An overview and analysis is presented regarding the epidemiology and consequences of physical inactivity and sedentary lifestyle, the main effects of isolation, the benefits of well prescribed, advised and performed exercise under various conditions, and chronic noncommunicable diseases and their interactions (Arabia, 2020).

\section{Situation of the Problem}

Real life and living requirements are imperative for a person, especially after the progress of life and after the mid-forty years or more. The program and organization of life must be prepared, including preparing and maintaining a fitness suit for these ages. Perhaps the difficulty of the matter in the presence of the health crisis caused by the Coronavirus may cause life difficult due to psychological, health and recreational pressures, and the researchers have seen through their professional and life experience that they are studying this problem scientifically for the purpose of reaching convincing results in exercising physical 
activity and sports, and according to Available areas in the home, subject to the home ban conditions. To identify the importance of physical activity and exercise for members of the research sample during the home ban during the Corona pandemic (Covid 2019) in 2020.

\section{METHOD}

The researchers used the descriptive approach method in their scientific studies. The research sample included (90) persons from the Algerian community, whose ages ranged from (45 to 55) years, a questionnaire was distributed to them through the Internet, due to the difficulty of meeting directly due to the lack of commitment to a home ban to avoid contracting Corona disease. The academic achievement for the members of the sample ranged from high school diploma to high school diploma holders.

\section{Material}

The questionnaire distributed to the sample included (10) different questions related to the aspects (health and psychological) and (recreational), and the answer was specific to some questions with the following options: (always - sometimes - never), and some questions specified the answer with (yes - no).

\section{Data Analyses}

The data were statistically processed through the following equation:

Percentage $=100 \times$ repetition $/$ sample number

\section{FINDINGS}

Analyzing the results:

Table 1: Are you bound by the home ban?

\begin{tabular}{ccc}
\hline \hline The answer & ITERATIONS & Percentage (\%) \\
\hline \hline Yes & 82 & 91.11 \\
No & 08 & 8.88 \\
Total & 90 & 100 \\
\hline \hline
\end{tabular}

It appears that the responses of the sample members related to the question of commitment to home prohibition, the number of repetitions of the answer was (Yes) (82) repetitions, while the percentage was $(91.11 \%)$ and compared to the answer with (No) that reached (08) iterations And with a percentage of $(8.88 \%)$, this means that the majority of the sample members were committed to the home ban for reasons related to their interpretation of the condition they are on because of the seriousness of Corona's disease (Covid 19) and perhaps cultural awareness prevented the rest of the sample's commitment and their weak awareness of the seriousness of the situation and considering that The sample is heterogeneous in terms of thought and academic achievement.

Table 2: Do you feel healthy and strong when engaging in physical activity and exercising at home during the ban period due to the Corona pandemic?

\begin{tabular}{ccc}
\hline \hline The answer & ITERATIONS & Percentage (\%) \\
\hline \hline Always & 86 & 95.55 \\
Sometimes & 04 & 4.44 \\
Permanently & 00 & 00 \\
Total & 90 & 100
\end{tabular}

It appears that the answers related to the feeling of health and strength of the members of the sample when practicing physical activity and practicing home sport during the ban period due to the Corona pandemic, the number of repetitions of the answer was (always) (86) repeats and a percentage of (95.55\%). As for the answer With (sometimes), the number of iterations reached (04) iterations and a percentage of $(4.44 \%)$, and the answer to (never) has got (00) iterations and this means they have no percentage (00) of the answers, 
which means that the majority of individuals The sample has a positive feeling for reasons related to their interpretation of the situation they are on because of the seriousness of Corona's disease (Covid 19) and perhaps cultural awareness prevented the commitment of the rest of the sample and their poor awareness of the gravity of the situation and considering that the sample is heterogeneous in terms of thought and academic achievement.

This means that the majority of individuals in the sample have a feeling that they have health and strength when practicing physical activity and exercising home sports due to the seriousness of Corona's disease (Covid 19), researchers believe that exercise generates a good feeling in the individual that he possesses health and physical strength and he must continue daily or in a timetable Organized for a specific number of days of the week, according to leisure time, taking into account appropriate nutrition, age and exercise intensity.

Table 3:Psychological security: Is there a feeling of lack of fear and a feeling of psychological reassurance about your health while practicing physical activity and practicing home sports during the period of prohibition under the Corona pandemic?.

\begin{tabular}{ccc}
\hline \hline The answer & ITERATIONS & Percentage \\
& & $(\%)$ \\
Always & 84 & 93.33 \\
Sometimes & 05 & 5.55 \\
Permanently & 01 & 1.11 \\
Total & 90 & 100 \\
\hline \hline
\end{tabular}

It appears that the responses of the sample members related to a feeling of lack of fear and a feeling of psychological reassurance after practicing physical activity and exercising at home during the period of prohibition under the Corona pandemic, the number of repetitions of the answer was (always) (84) repeats and a percentage of $(93.33 \%)$ As for the answer to (sometimes), the number of iterations reached (05) iterations and a percentage of $(5.55 \%)$, and the answer to (never) got (01) iterations and by a percentage of $(1.11 \%)$, meaning that the majority of individuals The sample have a feeling of lack of fear and a feeling of psychological reassurance about your health during practicing physical activity and exercising home sports during the period of prohibition under the Corona pandemic, and researchers see that exercise generates a good feeling in the individual that he possesses health and physical strength and he must continue daily or in an organized schedule for a specific number From days of the week according to leisure time, taking into account appropriate nutrition, age and exercise intensity, the US Department of Health recommends adults between the ages of 18 and 64 who exercise moderate physical activity on a regular basis.

Table 4: Do you have a feeling and a sense of self-confidence while practicing physical activity and exercising at home during the period of the ban under the Corona pandemic?

\begin{tabular}{ccc}
\hline \hline The answer & ITERATIONS & Percentage (\%) \\
\hline \hline Yes & 84 & 93.33 \\
No & 06 & 6.66 \\
Total & 90 & 100 \\
\hline \hline
\end{tabular}

It appears that the answers related to the feeling of the individuals of the sample feeling their self-confidence during practicing physical activity and practicing home sports during the period of the ban under the Corona pandemic, the number of repetitions of the answer was (Yes) (84) repetitions, while the percentage of the amount was (93.33\%) And compared to the answer to (no) that reached (06) repeatedly and by a percentage of $(6.66 \%)$, this is an indication that the majority of the sample members had a feeling of psychological satisfaction through their self-confidence, which generates a good health condition that contributes to strengthening their immune system and thus enjoying health It is good that they are in these ages (45-55) years, which creates an optimistic and coherent society and serves the public interest and for reasons that may relate to a wrong interpretation by the rest of the sample when they chose the word (no) to 
express their opinion of the situation they are in because of the seriousness of Corona's disease (Covid 19) Everyone is as rumored in the media and social media is now within reach of everyone.

Table 5: Is there a psychological improvement in the situation after the practice of physical activity and home exercise sports during the ban period under pandemic Corona?

\begin{tabular}{ccc}
\hline \hline The answer & ITERATIONS & Percentage (\%) \\
Yes & 87 & 96.66 \\
No & 03 & 3.33 \\
Total & 90 & 100 \\
\hline \hline
\end{tabular}

It appears that the answers related to the feeling of improvement in the psychological state among the members of the research sample after practicing physical activity and practicing home sport during the ban period under the Corona pandemic, the number of repetitions of the answer has reached (Yes) (87) repetitions, and the percentage of the amount was (96.66\%), and compared to the answer to "no", which reached (03) iteratively and with a percentage of (3.33\%), which indicates that the vast majority of respondents had a feeling of improvement in their psychological condition after frequent and many different news about Corona's disease and how dangerous it is to Humans.

Table 6: Is there a feeling of getting rid of thinking about worrying about the future while practicing physical activity and practicing home sports during the ban period under the Corona pandemic?

\begin{tabular}{ccc}
\hline \hline The answer & ITERATIONS & Percentage (\%) \\
\hline \hline Always & 81 & 90 \\
Sometimes & 09 & 10 \\
Permanently & 00 & 00 \\
Total & 90 & 100
\end{tabular}

It appears that the answers related to the feeling of the members of the sample to get rid of thinking with concern for the future after practicing physical activity and exercising home sports during the period of the ban under the Corona pandemic, the number of repetitions with (always) reached (81) repetitions and a percentage of (90\%) As for the answer to (sometimes), the number of iterations reached (09) iterations with a percentage of $(10 \%)$, and the answer to (never) got $(00)$ iterations and with a percentage of $(00 \%)$, that the individual feels anxious The increased risk of Corona disease has become evident and palpable to everyone, but the feeling of the members of the sample to get rid of anxiety over the future of their lives has decreased when they exercise, and this is because of their belief that it increases the body's immunity from this danger. Table 7:Did your appetite for food become good after engaging in physical activity and exercising at home during the ban period under the Corona pandemic?.

\begin{tabular}{ccc}
\hline \hline The answer & ITERATIONS & Percentage (\%) \\
\hline \hline Yes & 88 & 97.77 \\
No & 02 & 2.22 \\
Total & 90 & 100 \\
\hline \hline
\end{tabular}

It appears that the answers related to the appetite of individuals in the research sample for food after practicing physical activity and practicing home sport during the ban period under the Corona pandemic, the number of repetitions of the answer has reached (Yes) (88) repetitions, while the percentage of the amount was (97.77\%) And compared to the answer to (no), which reached (02) repeatedly and by a percentage of $(2.22 \%)$, that exercising regularly and in a manner suitable for the ages of the sample members has contributed significantly to retaining their appetite for eating and thus strengthening the body and preserving its being, which is hurt in The effectiveness of the immune system against diseases, including corona disease. 
Table 8: Is there a sense of moral relief during physical activity and exercising at home during the ban period under the Corona pandemic?.

\begin{tabular}{ccc}
\hline \hline The answer & ITERATIONS & Percentage (\%) \\
\hline \hline Always & 87 & 96.66 \\
Sometimes & 03 & 3.33 \\
Permanently & 00 & 00 \\
Total & 90 & 100 \\
\hline \hline
\end{tabular}

It appears that the answers related to the feeling of emotional satisfaction among the members of the sample after practicing physical activity and exercising at home during the ban period under the Corona pandemic The number of repeats with (always) (87) repeats and a percentage of (96.66\%), With (sometimes) the number of iterations reached (03) iterations with a percentage of (3.33\%), and the answer to (never) has got (00) iterations and with a percentage of $(00 \%)$, we conclude through the mentioned numbers that the vast majority For members of the sample, they have a positive feeling and high spirits after practicing physical and athletic activity, according to the researchers 'opinion that the increase in physical fitness through regular exercise contributes to creating a physical and health condition for the individual that enables him to check on his general health more than the inactive individual all the time, because the normal blood circulation of a person It continues in its proper form as a result of muscle contractions and contractions associated with the nature of aerobic and anaerobic exercises, which have the primary role in maintaining the heart rate in proportion to the age of the sample, and (Almadhkhori , 2019)states that all muscles in the body have the ability to contract, and therefore physical fitness plays an essential role in the health of Individuals.

Table 9:Do you feel the elimination of excess anxiety about the possibility of hatred after practicing physical activity and exercising at home during the period of prohibition under the Corona pandemic?.

\begin{tabular}{ccc}
\hline \hline The answer & ITERATIONS & Percentage (\%) \\
\hline \hline Yes & 77 & 85.55 \\
No & 13 & 14.44 \\
Total & 90 & 100 \\
\hline \hline
\end{tabular}

It appears that the answers related to eliminating excess anxiety by the possibility of hatred for members of the research sample after engaging in physical activity and exercising at home during the ban period under the Corona pandemic, the number of repetitions of the answer has reached (Yes) (77) repetitions and the percentage was Its amount (85.55\%), and compared to the answer to (no) which reached (13) iteratively and with a percentage of $(14.44 \%)$, it is clear from these percentages that the majority of the sample members have a positive feeling that their lives are not with that great risk as a result of the outbreak of Corona's disease And by their habit of exercising away from mixing with others in public places, it is considered an important factor and it is capable of removing or weakening the danger of corona against them.

Table 10: Do you feel that you have more control over your daily behaviors and emotions after engaging in physical activity and exercising at home during the ban period under the Corona pandemic?

\begin{tabular}{ccc}
\hline \hline The answer & ITERATIONS & Percentage (\%) \\
\hline \hline Yes & 76 & 84.44 \\
No & 14 & 15.55 \\
Total & 90 & 100 \\
\hline \hline
\end{tabular}

It appears that the answers related to controlling the daily actions and emotions of the research sample after practicing physical activity and practicing home sport during the ban period under the Corona pandemic, the number of repetitions of the answer has reached (Yes) (76) repetitions, while the percentage of the amount was (84.44\%), and compared to the answer to (no) that reached (14) iteratively and with a percentage of $(15.55 \%)$, perhaps in this axis of the study the percentage of answers in the positive direction decreased somewhat, despite the answer yes, the percentage was high, and the researchers suggest This slight decline here, may be due to the individual's previous normal nature, especially if we associate it with the ages of the sample members, because it is very difficult to change the condition of the individual and this age with a short period of experiences that may be new to some of them, and at the same time the answers are very logical and they represent an acceptable case very community members. 


\section{RESULT, DISCUSSION, AND SUGGESTIONS}

Cultural awareness was good for the individuals in the sample with the seriousness of Corona's disease. The majority of respondents have a feeling that they have health and strength when exercising physical activity and exercising home sports. Playing sports generates a good feeling for the individual that he possesses health and physical strength, and he has to continue daily or in an organized schedule for a specific number of days of the week and according to leisure times, taking into account appropriate nutrition, age and intensity of exercises. The US Department of Health recommends adults between the ages of 18 and 64 years of activity (WHO, 2020a). Most of the sample members had a feeling of psychological satisfaction through their self-confidence, which generates a good health condition that contributes to strengthening their immune system and thus enjoy good health and they are in these ages (45-55) years. Exercising regularly and in a manner appropriate to the ages of the sample, has contributed significantly to their appetite for eating, thus strengthening the body and maintaining its existence, which affects the effectiveness of the immune system against diseases, including corona disease. The high physical fitness through regular exercise contributes to creating a physical and health condition for the individual that enables him to check on his general health more than the inactive individual all the time, because the human's natural blood circulation continues in its proper form as a result of contractions and muscle contractions associated with the nature of aerobic and anaerobic exercises that have the primary role in Maintaining the heart rate in proportion to the age of the sample (Al-Mathkoori, 2019) states that all muscles in the body have the ability to contract, and therefore physical fitness plays an essential role in the health of individuals. Individuals at the age of (45-55) when practicing physical activity and exercising at home enjoy control of excessive emotions, which in the opposite case may weaken their immune system as a result of weak nerves and the circulatory system. When practicing physical activity and exercising at home during the ban period as a result of the consequences of Corona disease (Covid 19) is considered a state of recreational activity and a positive psychological state for the members of the research sample.

\section{REFERENCES}

Almadhkhori ,H.A. \& Al-Shammari, R. (2020). Healthy nutrition and the human musculoskeletal system. Moon Printing and Publishing,Iraq, Baghdad, ISBN: 9789922919256,p 123.

Almadhkhori,H.(2019). Biomechanics and kinetic analysis in sports. Moon Printing and Publishing, Iraq, Baghdad.

Arabia,J.J.M. (2020). Physical inactivity, exercise and pandemic COVID-19. Junio 9 (2).

Arlene, S. (2017). The top 10 benefits of regular exercise. Retrieved "www.healthline.com", 2018-8-3.

Bergland,A. \& Rydwik,E.(2018), The Importance of physical activity exercise among older people. Biomed Res Int , doi: $10.1155 / 2018 / 7856823$.

Booth,F.W., Roberts,C.K. \& Laye,M.J. (2012). Lack of exercise is a major cause of chronic diseases. Comprehensive Physiology, 2(2):1143-1211.

El-Khoury F., Cassou B., Charles M.-A. \& Dargent-Molina P(2013). The effect of fall prevention exercise programmes on fall induced injuries in community dwelling older adults: Systematic review and meta-analysis of randomised controlled trials. BMJ, 347, doi: 10.1136/bmj.f6234.f6234.

Flores-Tena, M.-J. (2019). El envejcimiento activo y la inteligencia emocional en las personas mayores. Revista de Ciencias y Orientación Familiar, 56, 125-137. ISSN 1130-8893.

Gillespie, L.D., Robertson, M., Gillespie W.J., Sherrington, C., Gates, S., Clemson, L.M. \& Lamb, S.E. (2012). Interventions for preventing falls in older people living in the community. Cochrane Database of Systematic Reviews, 9,DOI: 10.1002/14651858.CD007146.pub3

Greener,J., Douglas,F. \& Teijlingen,E. (2010). More of the same? Conflicting perspectives of obesity causation and intervention amongst overweight people, health professionals and policy makers. Social Science E Medicine , 70, (7), 1042-1049.

Nuñez-Gonzalez, José, et al.(2017). GS-005: Proyectos extensionistas comunitarios de la Facultad de Medicina de la Universidad del Zulia. Investigación Clínica, 58, (1). 
Scheiner,G. (2010). Sports and exercise: The ultimate challenge in blood sugar control.Retreived https://diatribe.org/sports-and-exercise-.

Tricco A. C., Thomas S. M., Veroniki A. A., et al(2017). Comparisons of interventions for preventing falls in older adults: A systematic review and meta-analysis. Journal of the American Medical Association, 318(17):1687-1699. doi: 10.1001/jama.2017.15006.

World Health Organization (2020). Estrategia mundial sobre régimen alimentario, actividad física y salud -Actividad física. https://www.who.int/dietphysicalactivity/pa/es . 